\title{
Qualidade de vida e estado nutricional de idosos institucionalizados e não
}

\section{institucionalizados}

\author{
Quality of life and nutritional status of institutionalized and non-institutionalized elderly \\ Calidad de vida y estado nutricional de ancianos institucionalizados y no institucionalizados
}

Tainá Facchini

ORCID: https://orcid.org/0000-0001-8507-126X Universidade do Vale do Taquari, Brasil

E-mail: taina.facchini@universo.univates.br

Fernanda Scherer Adami

ORCID: https://orcid.org/0000-0002-2785-4685 Universidade do Vale do Taquari, Brasil

E-mail: fernandascherer@univates.br

Nathascha dos Santos Trindade

ORCID: https://orcid.org/0000-0002-5984-2793 Universidade do Vale do Taquari, Brasil

E-mail: nathascha.trindade@universo.univates.br

Jéssica Carina Führ

ORCID: https://orcid.org/0000-0003-1753-444X Universidade do Vale do Taquari, Brasil

E-mail: jefuhr@universo.univates.br

Janine Maria Lavall

ORCID: https://orcid.org/0000-0002-9370-6074

Universidade do Vale do Taquari, Brasil

E-mail: janinepeiter@universo.univates.br

Fabiani Cristina Lassen Delazzeri

ORCID: https://orcid.org/0000-0003-1903-0106 Universidade do Vale do Taquari, Brasil

E-mail: fcldelazzeri@universo.univates.br

Simara Rufatto Conde

ORCID: https://orcid.org/0000-0003-1531-7433 Universidade do Vale do Taquari, Brasil

E-mail: simararufatto@univates.br

Juliana Paula Bruch Bertani

ORCID: https://orcid.org/0000-0002-2427-2858 Universidade do Vale do Taquari, Brasil E-mail: julianapb@univates.br

Patricia Fassina

ORCID: https://orcid.org/0000-0001-5467-2505 Universidade do Vale do Taquari, Brasil E-mail: patriciafassina@univates.br

\begin{abstract}
Resumo
Objetivo: Associar os domínios de qualidade de vida com o estado nutricional e idade de idosos não institucionalizados e residentes em Instituições de Longa Permanência para Idosos (ILPIs). Metodologia: Trata-se de um estudo retrospectivo, transversal, de natureza quantitativa, com 105 idosos não institucionalizados atendidos em uma Estratégia de Saúde da Família (ESF), município de Lajeado-RS, e 245 idosos institucionalizados residentes em ILPIs públicas e privadas, no Rio Grande do Sul. Utilizou-se o Questionário de Avaliação de Qualidade de Vida The World Health Organization Quality of Life - WHOQOL-bref composto de 26 questões, de escala hedônica de 1 a 5. Realizou-se aferição do peso e estatura, para determinar o índice de massa corporal (IMC) e classificar o estado nutricional conforme Organização Pan-Americana da Saúde (2002). Consideraram-se significativos os resultados a um nível de significância máxima de 5\%. Resultados: O maior escore de qualidade de vida dos idosos observou-se no

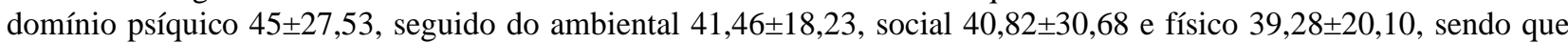
os domínios de qualidade de vida foram significativamente superiores entre os idosos com 60 a 69 anos (p $\leq 0,01)$, com estado nutricional classificados como obesidade $(p \leq 0,01)$ e os não institucionalizados quando comparados com os institucionalizados. Conclusão: A percepção dos domínios de qualidade de vida dos idosos estudados foi maior no
\end{abstract}


domínio psíquico e menor no físico. Os idosos mais jovens, com obesidade e não institucionalizados demonstraram uma melhor percepção da qualidade de vida em todos os domínios.

Palavras-chave: Idosos; Qualidade de vida; Estratégia Saúde da Família; Institucionalização.

\begin{abstract}
Objective: To associate the domains of quality of life with institutionalized and non-institutionalized elders' nutritional state and age in Long Stay Institutions for the Elderly. Methodology: This study is retrospective, transversal, and quantitative developed with 105 non-institutionalized assisted by a Family Health Strategy (FHS) in Lajeado and 245 institutionalized elders, who live in private and public Long Stay Institutions for the Elderly (LSIEs) both in Rio Grande do Sul. We used the World Health Organization Quality of Life Questionnaire (WHOQOL-bref), which is composed by 26 questions with a hedonic scale from 1 to 5 . We assessed the elder's weight and height in order to determine the body mass index (BMI) and to classify their nutritional state according to Pan American Health Organization (2002). We consider as significant the results with a significance level of 5\%. Results: The higher score of elders' quality of life was noticed in the psychic domain $45 \pm 27,53$, followed by the environmental $41,46 \pm 18,23$, social $40,82 \pm 30,68$ and physical $39,28 \pm 20,10$ being the domains of quality of life significantly superior among the elders from 60 to 90 years $(p \leq 0,01)$, the ones with nutritional state classified as obesity $(p \leq 0,01)$ and the noninstitutionalized when compared to the institutionalized ones. Conclusion: The perception of the studied domains of quality of life was higher in the psychic than in the physical domain. The younger elders, the ones with obesity and the non-institutionalized showed a better perception of quality of life in all the domains.
\end{abstract}

Keywords: Elders; Quality of life; Family Health Strategy; Institutionalization.

\title{
Resumen
}

Objetivo: Asociar los dominios de calidad de vida con el estado nutricional y edad de ancianos no institucionalizados y residentes de Instituciones de Larga Permanencia para Ancianos. Metodología: Este estudio es retrospectivo, transversal y cuantitativo, desarrollado con 105 ancianos no institucionalizados atendidos en una Estrategia de Salud de la Familia (ESF), en Lajeado, y 245 ancianos institucionalizados residentes en Instituciones de Larga Permanencia para Ancianos (ILPAs) públicas y privadas, ambas en Rio Grande do Sul. Se utilizó el Cuestionario de Evaluación de Calidad de Vida The World Health Organization Quality of Life - WHOQOL-bref, compuesto por 26 cuestiones, de escala hedónica de 1 a 5. Se realizó la verificación del peso y de la estatura para determinar el índice de amsa corporal (IMC) y clasificar el estado nutricional conforme la Organización Panamericana de Salud (2002). Fueron considerados significativos los resultados a un nivel de significancia máxima de 5\%. Resultados: La puntuación más alta de calidad de vida de los ancianos fue observada en el dominio psíquico 45 $\pm 27,53$, seguido del ambiental $41,46 \pm 18,23$, social 40,82 $\pm 30,68$ y físico 39,28 $\pm 20,10$, siendo que los dominios de calidad de vida fueron significativamente superiores entre $\operatorname{los}$ ancianos con 60 a 90 años $(\mathrm{p} \leq 0,01)$, aquellos con estado nutricional clasificados como obesidad $(\mathrm{p} \leq 0,01)$ y los no institucionalizados, cuando comparados con los institucionalizados. Conclusión: La percepción de los dominios de calidad de vida de los ancianos estudiados fue más grande en el dominio psíquico y menor en el físico. Los ancianos más jóvenes, con obesidad y no institucionalizados demostraron una mejor percepción de la calidad de vida en todos los dominios.

Palabras-clave: Ancianos; Calidad de vida; Estrategia de Salud Familiar; Institucionalización.

\section{Introdução}

O envelhecimento populacional vem crescendo gradativamente no Brasil e está associado a importantes alterações demográficas, sociais e econômicas que acarretam a formulação e implementação de políticas públicas no âmbito da proteção social ao idoso (Souza, 2015). Envelhecer, para alguns idosos, significa aproveitar as oportunidades que surgem ao longo da vida para alcançar e manter a saúde, além de ter uma ocupação rica em significado, relações sociais, novas habilidades, conhecimento e necessidades materiais (Cilb, 2015). Assim, um ambiente de boas condições de bem-estar e qualidade de vida aumentam a expectativa de vida do idoso (Ine, 2019).

Espera-se que até 2040 tenhamos 56,6 milhões de idosos brasileiros, ou seja, 27,5\% da população no geral (Kanso, 2013). Os dados publicados pelo Instituto Brasileiro de Geografia e Estatística (IBGE), em 2018, demonstraram que a população brasileira possui 210.246.098 milhões de pessoas, destas 30,2 milhões com 60 anos ou mais. Nos últimos sete anos a população idosa aumentou 18\%, sendo as mulheres 16,9 milhões (56\%) e os homens 13,3 milhões (44\%) (IBGE, 2018).

Com o aumento do número de idosos e da sua expectativa de vida, é importante garantir uma melhor qualidade de vida entre esta população, contemplando a saúde, o trabalho, as condições de moradia, as relações sociais e a autonomia (Silva 
et al., 2017). O estado nutricional representa uma condição importante para os determinantes de saúde e doença, visto que diagnósticos nutricionais extremos, como a desnutrição e a obesidade, relacionam-se a múltiplas morbidades em idosos, sendo a avaliação nutricional uma importante ferramenta de prevenção no contexto do envelhecimento (Ferreira et al., 2020).

Preocupar-se com a qualidade de vida na terceira idade desde o princípio é uma forma de ampliar a capacidade do próprio organismo. Os idosos percebem a sua qualidade de vida de diferentes formas, principalmente, por esse ser um período de grandes mudanças, limitações e preocupações (Oliveira et al., 2017). A percepção de qualidade de vida dos idosos institucionalizados apresenta-se inferior aos não institucionalizados, possivelmente devido a fatores que causam a institucionalização, tais como idade, sexo, nível de escolaridade, estilo de vida, autonomia e participação social. Entre os domínios avaliados nos questionários de qualidade de vida a autonomia e aspectos ambientais normalmente são os que menos satisfazem os idosos institucionalizados (Moreira et al., 2016).

Portanto, o objetivo deste estudo foi associar os domínios de qualidade de vida com o estado nutricional e idade de idosos não institucionalizados e residentes em Instituições de Longa Permanência para Idosos.

\section{Metodologia}

Trata-se de um estudo retrospectivo, transversal, de natureza quantitativa, no qual coletaram-se e analisaram-se dados quantitativos de caráter observacional (Pereira et al., 2018), realizado a partir de dois estudos aprovados pelo Comitê de Ética e Pesquisa da Universidade do Vale do Taquari (Univates). Um deles foi aprovado sob protocolo número 3.380.577, com idosos não institucionalizados, atendidos em uma Estratégia de Saúde da Família (ESF) do município de Lajeado, localizada no Estado do Rio Grande do Sul (RS), no período de julho a setembro de 2019 e o outro sob o número 2.303.383, com idosos institucionalizados de nove Instituições de Longa Permanência para Idosos (ILPIS), no período de 2017 a 2020.

Avaliou-se o estado nutricional através do peso atual do idoso que foi aferido através de uma balança digital portátil da marca Omron® com graduação mínima de $100 \mathrm{~g}$ e capacidade máxima de $150 \mathrm{~kg}$, o indivíduo estava descalço, com o mínimo de roupa possível, em posição ortostática, posicionada no centro do equipamento, ereto, com os pés juntos e os braços estendidos ao longo do corpo. A altura foi aferida em posição ortostática, com estadiômetro portátil marca Sanny Profissional ${ }^{\circledR}$ com precisão de $0,1 \mathrm{~cm}$ e extensão máxima de dois metros, fixado na parede, estando o idoso descalço, com a cabeça livre de adereços, ereto, com os braços estendidos ao longo do corpo, as pernas paralelas formando um ângulo reto com os pés e a cabeça erguida, olhando para um ponto fixo na altura dos olhos (BRASIL, 2011). Classificou-se o estado nutricional dos idosos através do índice de massa corporal (IMC), a partir das referências da Organização Pan-Americana da Saúde (OPAS, 2002).

O Idoso também respondeu o Instrumento de Avaliação de Qualidade de Vida The World Health Organization Quality of Life - WHOQOL-bref composto de 26 questões, de escala hedônica de 1 a 5. Destas, 24 questões são divididas em quatro domínios de qualidade de vida: capacidade física, bem-estar psicológico, relações sociais e meio-ambiente. As duas questões restantes são gerais, referentes à qualidade de vida. Ainda categorizou-se a idade dos idosos entre 60 a 69 anos, 70 a 79 e mais de 80 anos.

Incluíram-se neste estudo os dados registrados referentes aos idosos com idade igual ou superior a 60 anos, frequentadores da ESF e ILPIS, de ambos os sexos, lúcidos, orientados, que consentiram em participar da pesquisa após a assinatura do Termo de Consentimento Livre e Esclarecido (TCLE), totalizando 350 idosos.

A análise estatística realizou-se por meio dos testes não-paramétricos de Kruskal-Wallis, teste não-paramétrico de Mann-whitney e análise de Correlação. Os resultados foram considerados significativos a um nível de significância máximo de $5 \%(\mathrm{p} \leq 0,05)$ e o software utilizado para esta análise foi o Epi Info 7.2. 


\section{Resultados}

Do total de idosos participantes do estudo, $70 \%$ (245) residiam em ILPIs e 30\% (105) vivem sozinhos ou com familiares, 34,3\% (120) eram do sexo masculino e 65,7\% (230) do feminino, 37,1\% (130) estavam com mais de 80 anos, 23,3\% (113) de 70 a 79 anos e 30,6\% (107) de 60 a 69 anos. Em relação ao estado nutricional, a maioria classificou-se com eutrofia, 39,1\% (137), seguido de obesidade, 28,3\% (99), baixo peso 17,4\% (61) e sobrepeso 15,1\% (53).

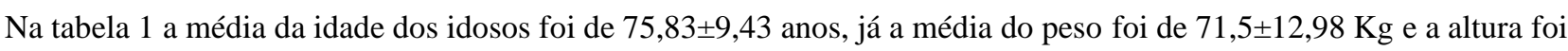

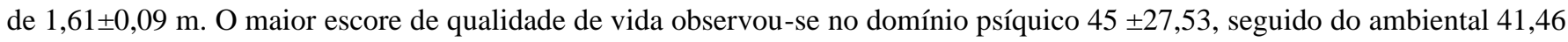
$\pm 18,23$, social 40,82 $\pm 30,68$ e do físico. (Tabela 1 )

Tabela 1. Caracterização da idade, peso, altura, Índice de Massa Corporal e domínios de qualidade de vida dos idosos institucionalizados e não institucionalizados.

\begin{tabular}{cllll}
\hline Variáveis & Mínimo & Máximo & Média & Desvio Padrão \\
\hline Idade & 60,00 & 96,00 & 75,83 & 9,43 \\
Peso $(\mathrm{Kg})$ & 37,00 & 129,80 & 71,50 & 12,98 \\
Altura (m) & 1,38 & 1,82 & 1,61 & 0,09 \\
Índice de Massa Corporal $\mathrm{Kg} / \mathrm{m}^{2}$ & 16,44 & 44,68 & 27,60 & 5,03 \\
Domínio Físico & 7,14 & 89,30 & 39,28 & 20,10 \\
Domínio Psíquico & 4,17 & 95,80 & 45,00 & 27,53 \\
Domínio Social & 0,00 & 100,00 & 40,82 & 30,68 \\
Domínio Ambiental & 9,38 & 84,40 & 41,46 & 18,23 \\
\hline
\end{tabular}

Fonte: Autores.

Na Tabela 2 observou-se que os escores dos domínios físico, psíquico, social e ambiental foram significativamente superior $(\mathrm{p} \leq 0,01)$ entre os idosos não institucionalizados em relação aos institucionalizados.

Tabela 2. Comparação dos escores dos domínios de qualidade de vida dos idosos institucionalizados e não institucionalizados.

\begin{tabular}{|c|c|c|c|c|c|}
\hline Domínio & Idoso & $\mathrm{n}$ & Média & Desvio Padrão & $\mathrm{P}$ \\
\hline \multirow[t]{2}{*}{ Físico } & Institucionalizados & 245 & 29,10 & 12,02 & $\mathrm{p} \leq 0,01$ \\
\hline & Não Institucionalizado & 105 & 63,03 & 14,25 & \\
\hline \multirow[t]{2}{*}{ Psíquico } & Institucionalizados & 245 & 29,54 & 16,15 & $\mathrm{p} \leq 0,01$ \\
\hline & Não Institucionalizado & 105 & 81,07 & 7,42 & \\
\hline \multirow[t]{2}{*}{ Social } & Institucionalizados & 245 & 24,63 & 20,23 & $\mathrm{p} \leq 0,01$ \\
\hline & Não Institucionalizado & 105 & 78,59 & 11,93 & \\
\hline \multirow[t]{2}{*}{ Ambiental } & Institucionalizados & 245 & 32,18 & 12,15 & $\mathrm{p} \leq 0,01$ \\
\hline & Não Institucionalizado & 105 & 63,12 & 9,58 & \\
\hline
\end{tabular}

Teste não-paramétrico de Mann-Whitney. Fonte: Autores.

Verificou-se que os escores dos domínios de qualidade de vida foram significativamente superiores entre os idosos com 60 a 69 anos, seguidos dos idosos com idade entre 70 a 79 anos, e por fim, com menores escores estão os idosos com 80 anos ou mais $(\mathrm{p} \leq 0,01)$ (Tabela 3$)$. 
Tabela 3. Comparação dos escores dos domínios de qualidade de vida entre as faixas de idade dos idosos.

\begin{tabular}{llllll}
\hline Domínio & Idade (anos) & $\mathrm{n}$ & Média & Desvio Padrão & $\mathrm{P}$ \\
\hline Físico & $60-69$ & 107 & 50,41 & 21,78 & 19,85 \\
& $70-79$ & 113 & 39,00 & 13,36 & $\mathrm{p} \leq 0,01$ \\
& 80 ou mais & 130 & 30,37 & 28,96 & $\mathrm{p} \leq 0,01$ \\
\hline Psíquico & $60-69$ & 107 & 61,16 & 26,55 & 19,64 \\
& $70-79$ & 113 & 43,46 & 31,84 & $\mathrm{p} \leq 0,01$ \\
& 80 ou mais & 130 & 33,04 & 31,46 & 22,70 \\
\hline Social & $60-69$ & 107 & 56,15 & 18,38 \\
& $70-79$ & 113 & 40,07 & 18,03 \\
& 80 ou mais & 130 & 28,85 & 13,57 \\
\hline
\end{tabular}

Teste não-paramétrico de Kruskal-Wallis. Fonte: Autores.

$\mathrm{Na}$ Tabela 4 observou-se que todos os escores dos domínios de qualidade de vida foram significativamente superiores $(\mathrm{p} \leq 0,01)$, entre os idosos classificados com obesidade, entretanto as classificações de baixo peso, eutrofia e sobrepeso não foram observadas diferenças significativas.

Tabela 4. Comparação dos escores dos domínios de qualidade de vida com as classificações de estado nutricional dos idosos.

\begin{tabular}{|c|c|c|c|c|c|}
\hline Domínio & IMC & $\mathrm{N}$ & Média & Desvio Padrão & $\mathrm{p}$ \\
\hline \multirow[t]{4}{*}{ Físico } & Baixo peso & 61 & 35,10 & 16,06 & \multirow[t]{4}{*}{$\mathrm{p} \leq 0,01$} \\
\hline & Eutrofia & 137 & 35,31 & 17,84 & \\
\hline & Sobrepeso & 53 & 36,01 & 21,05 & \\
\hline & Obesidade & 99 & 49,10 & 21,63 & \\
\hline \multirow[t]{4}{*}{ Psíquico } & Baixo peso & 61 & 40,26 & 23,40 & \multirow[t]{4}{*}{$\mathrm{p} \leq 0,01$} \\
\hline & Eutrofia & 137 & 39,26 & 25,49 & \\
\hline & Sobrepeso & 53 & 38,29 & 25,78 & \\
\hline & Obesidade & 99 & 59,45 & 28,53 & \\
\hline \multirow[t]{4}{*}{ Social } & Baixo peso & 61 & 32,25 & 24,89 & \multirow[t]{4}{*}{$\mathrm{p} \leq 0,01$} \\
\hline & Eutrofia & 137 & 35,35 & 29,46 & \\
\hline & Sobrepeso & 53 & 33,13 & 28,29 & \\
\hline & Obesidade & 99 & 57,79 & 30,57 & \\
\hline \multirow[t]{4}{*}{ Ambiental } & Baixo peso & 61 & 39,48 & 16,55 & \multirow[t]{4}{*}{$\mathrm{p} \leq 0,01$} \\
\hline & Eutrofia & 137 & 38,83 & 16,78 & \\
\hline & Sobrepeso & 53 & 36,00 & 17,88 & \\
\hline & Obesidade & 99 & 49,25 & 19,12 & \\
\hline
\end{tabular}

Teste não-paramétrico de Kruskal-Wallis. Fonte: Autores.

A idade dos idosos demonstrou correlações inversas e negativas com os escores dos domínios de qualidade de vida, indicando que quanto menor a idade do idoso maior é o valor do escore para os domínios. Enquanto o IMC apresentou correlações diretas e positivas, indicando que quanto maior o IMC do idoso maior é o valor do escore para os domínios $(\mathrm{p} \leq 0,01)$ (Tabela 5). 
Tabela 5. Correlação dos escores dos domínios de qualidade de vida com idade e Índice de Massa Corporal dos idosos.

\begin{tabular}{lllll}
\hline Domínio & Idade & \multicolumn{3}{l}{$\mathrm{IMC} \mathrm{Kg} / \mathrm{m}^{2}$} \\
\cline { 2 - 5 } & $\mathrm{R}$ & $\mathrm{P}$ & $\mathrm{r}$ & $\mathrm{P}$ \\
\hline Físico & $-0,447$ & $\mathrm{p} \leq 0,01$ & 0,291 & $\mathrm{p} \leq 0,01$ \\
Psíquico & $-0,445$ & $\mathrm{p} \leq 0,01$ & 0,302 & $\mathrm{p} \leq 0,01$ \\
Social & $-0,395$ & $\mathrm{p} \leq 0,01$ & 0,327 & $\mathrm{p} \leq 0,01$ \\
Ambiental & $-0,435$ & $\mathrm{p} \leq 0,01$ & 0,222 & $\mathrm{p} \leq 0,01$ \\
\hline
\end{tabular}

Análise de Correlação. Legenda: IMC: Índice de Massa Corporal. Fonte: Autores.

\section{Discussão}

Apesar da maioria dos idosos do presente estudo ter apresentado eutrofia, é importante destacar que 17,4\% (n=61) estavam em baixo peso e 28,3\% (n=99) em obesidade. Já no estudo de Barros et al. (2018), que avaliou os idosos institucionalizados e não institucionalizados de Montes Claros-MG, observou-se 30,6\% ( $\mathrm{n}=19$ ) com baixo peso e $22,6 \%$ ( $n=14)$ em obesidade, assim como Figueiredo et al. (2017) que verificaram que idosos institucionalizados e não institucionalizados de Agudos-SP, 22,63\% $(n=8)$ em baixo peso e 25,7\% $(n=9)$ com obesidade, resultados superiores em relação ao baixo peso e inferiores a obesidade quando comparados ao presente estudo.

O baixo peso em idosos está associado à incapacidade e dependência funcional, proveniente da sarcopenia e perda de força muscular, decorrentes do processo de envelhecimento, que contribuem para a inatividade física, reduzindo a capacidade funcional. Já o peso elevado além de contribuir para a incapacidade funcional, aumentando o risco de quedas, causa instabilidade corporal e corrobora para o agravamento de doenças crônicas degenerativas como Diabetes Mellitus, doenças cardiovasculares, hipertensão arterial e reumatismo, presentes na idade avançada (Santos et al., 2018).

Os escores dos domínios de qualidade de vida foram significativamente superiores entre os idosos classificados com obesidade, em relação aos com baixo peso, eutrofia e sobrepeso, resultado confirmado também na análise da relação das médias dos escores de qualidade de vida com o IMC, indicando que quanto maior o IMC do idoso maior foi o valor do escore para os domínios. Resultado diferente encontrado no estudo de Adami, Feil e Bosco (2015) com 156 idosos, utilizando o Whoqol-bref, onde indivíduos com IMC elevado, ou seja, em sobrepeso e obesidade, apresentaram médias dos escores de qualidade de vida menores que os demais.

A qualidade de vida muda de indivíduo para indivíduo de acordo com suas percepções, ações, desejos, anseios, peso e idade, o que pode explicar diferentes percepções entre os domínios de qualidade de vida (Souza et al., 2018). O excesso de massa corporal representa carga adicional na estrutura músculo esquelética de indivíduos obesos, levando a possíveis adaptações favoráveis na massa muscular, massa óssea e na força muscular, situação que pode estar relacionada com o aumento das médias dos escores de qualidade de vida nos domínios dos entrevistados com excesso de peso do presente estudo (Tavares et al., 2018 e Pillat et al., 2020).

O atual estudo demonstrou que a maior média dos escores dos domínios de qualidade de vida entre a totalidade dos idosos foi de 45,00 ( $\pm 27,53)$ no psíquico, seguido do domínio ambiental 41,46 ( $\pm 18,23), 40,82( \pm 30,68)$, social, e por fim, o físico 39,28 ( $\pm 20,10)$. Diferente de Coelho et. al (2021), que observaram no estudo realizado com idosos institucionalizados e não institucionalizados a maior média dos escores no domínio social, seguido do domínio psíquico, ambiental, e por fim, o físico. Em ambos os estudos supracitados, com a presença de idosos institucionalizados e não institucionalizados na amostra, observou-se uma menor média no escores do domínio físico, que pode estar relacionado com um menor desempenho físico, baixa escolaridade, perda da auto estima, características comuns entre estes idosos, podendo aumentar as chances de desenvolver patologias que causam decadências físicas e psicológicas mais expressivas (Costa et al., 2021). 
Entre os idosos não institucionalizados quando comparados aos institucionalizados os escores dos domínios físicos, psíquico, social e ambiental da qualidade de vida dos idosos foram significativamente superiores, resultado semelhante aos observados por Dias e Ribeiro (2018) ( $<<0,01)$ e diferente do estudo de Costa et. al (2018) que constataram que os grupos apresentaram valores similares nos domínios da qualidade de vida ( $>0,05)$. Diante disso, a institucionalização pode constituirse de uma situação em que o idoso se sente isolado de seu convívio social com membros da família, bem como dos problemas sociais externos. Essas rupturas afetam negativamente a satisfação com a qualidade de vida do idoso, que podem desencadear doenças crônicas não transmissíveis. Por isso, atividades recreativas em ILPIs auxiliam a aumentar as relações sociais destes idosos, por meio da interação com outras pessoas, que consequentemente constrói uma rede de apoio social e garante uma melhor qualidade de vida (Daniel et al., 2019; Uyeno et al., 2016).

No atual estudo, os escores dos domínios de qualidade de vida da totalidade dos idosos com 60 a 69 anos foi significativamente superior, seguido pelos com 70 a 79 anos, e por fim, os com 80 anos ou mais, ou seja, quanto menor a idade do idoso significativamente maior foi o valor do escore para todos domínios de qualidade de vida, resultado semelhante encontrado por Nogueira et. al (2016), em seu estudo no estado de Paraíba e ao estudo de Bocchi e Adami (2017) realizado no município de Doutor Ricardo- RS, que observou-se uma correlação inversa e significativa apenas entre a idade e o domínio físico e social $(\mathrm{p}=0,001)$. Pode-se dizer que os idosos mais jovens têm uma qualidade de vida melhor que os mais velhos, pois eles têm mais disposição para realização de atividade física, consequentemente melhorando a saúde psicológica além de prevenir doenças (Confortin et al., 2016).

Os idosos mais jovens têm como aspecto positivo o sentimento de felicidade, assim, os cuidados com o corpo e com a mente garantem uma visão positiva sobre a própria vida para quem está na terceira idade (Figueiredo et al., 2017). Já entre os idosos mais velhos o isolamento social é mais comum tendo menos contato com amigos e até família. É frequente que, com a saída dos filhos de casa e até com as mudanças em instituições de longa permanência ou doenças de amigos, os idosos fiquem solitários (Ferreira et al., 2018). Além destas questões emocionais e psicológicas, durante o envelhecimento também ocorrem mudanças físicas como a perda de 2 a 3\% da massa corporal magra por cada década, aumentando as chances da ocorrência da sarcopenia, contribuindo para a diminuição da força muscular, alterações do modo de andar, equilíbrio e perda da função física. A taxa metabólica de repouso diminui aproximadamente 15 a $20 \%$ durante a vida, o que diminui as necessidades de energia, devido a esta diminuição de massa muscular, aumento da gordura corporal e um estilo de vida mais sedentário, aumentando o risco de obesidade no idoso (Miranda et al., 2016).

Como limitação deste estudo é necessário destacar que os resultados obtidos podem não ser representativos para todos os idosos, uma vez que a amostra estudada foi composta por idosos residentes em ILPI do Vale do Taquari e Serra do RS, e idosos não institucionalizados de um município do Vale do Taquari. Portanto, percebe-se a necessidade de mais estudos com o intuito de promover saúde, qualidade de vida e bem-estar da população idosa. Além disso, o estudo não levou em consideração as patologias presentes na população estudada que podem interferir na qualidade de vida de idosos.

\section{Conclusão}

Concluiu-se que a percepção de qualidade de vida na amostra estudada foi melhor no domínio psíquico, seguido do domínio ambiental, social, e por fim, o físico. Os idosos não institucionalizados apresentavam escores dos domínios da qualidade de vida significativamente superior, quando comparados aos institucionalizados. Entre o total de idosos, a maioria apresentou eutrofia, seguido de obesidade e baixo peso, mas os classificados como obesos apresentaram escores significativamente superiores às demais classificações do estado nutricional. A relação entre o IMC e os escores de qualidade de vida foi significativamente positiva, enquanto a relação da idade com os escores foi significativamente negativa. Os idosos 
com 80 anos ou mais demonstraram escores significativamente menores para os domínios quando comparados às demais faixas etárias. Sugere-se que novas pesquisas sejam desenvolvidas para comparar a qualidade de vida e o estado nutricional dos idosos institucionalizados com os não institucionalizados, visando intervenções mais eficazes para cada população.

\section{Referências}

Adami, F. S., Feil, C. C., \& Bosco, S. M. (2015) Estado Nutricional Relacionado a Qualidade de Vida em Idosos. RBCEH, 12(1), 28-40.

Barros, M. C., Dias, B. N., Dias, J.; Barros, T. C., Gonçalves, J. T. T., \& Oliveira, M. V. M. (2018) Avaliação nutricional em idosos institucionalizados e não institucionalizados em Montes Claros/MG. Rev. Temas em Saúde, 18 (3), 12-26.

Bocchi, A \& Adami, F. S. (2017) Relação da qualidade de vida com o estado nutricional e faixa etária de idosos. $R B C E H, 14(1), 44-5$.

Cardozo, N. R., Duval, P. A., Cascais, A. M., Silva, A. E. R., \& Orlandi, S. P. (2017) Estado nutricional de idosos atendidos por unidades de saúde da família na cidade de Pelotas-RS. BRASPEN J, 32(1), 94-98.

Centro Internacional de Longevidade Brasil CILB. (2015) Envelhecimento Ativo: Um Marco Político em Resposta à Revolução da Longevidade. Centro Internacional de Longevidade Brasil. CILB. E-book.

Coelho, H. S., Sales, B. C. M., Soares, L. L., \& Júnior, R. J. M. (2020) Capacidade funcional, indicativos de sarcopenia, risco de quedas e qualidade de vida entre idosos institucionalizados, idosos praticantes não praticantes de exercício físico. Revista Científica UNIFAGOC. Caderno Multidisciplinar, 5(2), 24-36.

Confortin C. S. (2016) Atividades no lazer e Qualidade de vida de Idosos de um Programa de Extensão Universitária em Florianópolis (SC). Bras Ativ Fis Saúde, 19(4), 494-503.

Costa, F. R., Rodrigues, F. M., Prudente, C. O. M., \& Sousa, I. F. (2018) Qualidade de vida de idosos participantes e não participantes de programas públicos de exercícios físicos. Rev. Bras. Geriatr. Gerontol. Rio de Janeiro, 21(1), 24-34.

Costa, T. N. M., Nieto, J. P. de S., Morikawa, L. S., Araújo, A. V. S. de, Cardoso, A. A. M., Mafra, B. G., Eiró, M., \& Costa, V. O. da. (2021). Análise do Mini Exame do estado mental de Folstein em idosos institucionalizados e não institucionalizados/ Analysis of Folstein's Mini State examination in institutionalized and non institutionalized elderly people. Brazilian Journal of Health Review, 4(2), 8319-8336.

Daniel, F. S., Brites, A. P., Monteiro, R. \& Vicente, H. T. (2019) De "lar" abominado a estimado (ou tolerado): reconfiguração das representações sobre institucionalização. Saúde e Sociedade, 28(4), 2014-228.

Dias, E. N. \& Ribeiro, J. L. P. (2018) Qualidade de vida: comparação entre os idosos na comunidade e institucionalizados. Revista Kairós — Gerontologia, 21(1), 37-54.

Ferreira, L. C., Meireles, J. F. F., \& Ferreira, M. E. C. (2018) Avaliação do estilo e qualidade de vida em idosos: uma revisão de literatura. Rev. Bras. Geriatr. Gerontol, 21(5), 639-651.

Figueiredo, A. M., Damasceno, R. J., Damiance, P. M., Caldana, M. D. L. \& Bastos, J. R. D. M. (2017) Qualidade de vida e risco de desenvolver diabetes em idosos hipertensos institucionalizados e não institucionalizados do interior do estado de São Paulo. Saber Científico, 6(1), 38-47.

Godoy, A. R. \& Adami, F. S. (2019) Estado nutricional e qualidade de vida em adultos e idosos com depressão. Rev. Brasileira de Promoção à Saúde 32(7351), 1-12.

Instituto Brasileiro de Geografia e Estatística - IBGE. (2018) Número de idosos cresce $18 \%$ em 5 anos e ultrapassa 30 milhões em 2017. Rio de Janeiro, RJ: IBGE. /ibgeoficial/posts/2135841053109399.

Instituto Nacional de Estatística. (2019) Tábuas de Mortalidade em Portugal. Lisboa, PT: INE. Recuperado em: https://tinyurl.com/3wch7h6j.

Kanso, S. (2013) Processo de envelhecimento populacional - um panorama mundial. In: Workshop de Análise Ergônomica do Trabalho, VI.,Encontro Mineiro de Estudos em Ergonomia, III, Simpósio do Programa Tutorial em Economia Doméstica, VIII., Anais [...]. Viçosa, MG, UFV, p.1-23. Recuperado em: http://www.workshop-ded.ufv.br/wp-content/uploads/Solange-Kanso.pdf.

Miranda, G. M. D., Mendes, A. C. G.; \& Silva, A. L. A. (2016) O envelhecimento populacional brasileiro: desafios e consequências sociais atuais e futuras. Rev. Bras. Geriatr. Gerontol, Rio de Janeiro, 19(3), 507-519.

Nogueira, M. F., Lima, A. A., Trigueiro, J. S., Torquato, I. M. B.; Henriques, M. E. R. O., \& Alves, M. S. C. F. (2016) Comparando a qualidade de vida de idosos institucionalizados e não institucionalizados. Rev enferm UERJ, Rio de Janeiro, 24(5), 28-35.

Oliveira, B.C., Barbosa N. M., Lima M. S., Guerra H. S., Neves C. M., \& Avelar J. B. (2017) Avaliação da qualidade de vida em idosos da comunidade. Rev Bras Promoç Saúde, 30(3):1-10.

Organização Pan-Americano da Saúde-OPAS. IMC idosos. OPAS, 2002. Recuperado em: http://www.paho.org/bra.

Pereira, A. S., Shitsuka, D. M., Parreira, F. J. \& Shitsuka, R. (2018). Metodologia da pesquisa científica. [e-book]. RS: UAB/NTE/UFSM. Recuperado de: em https://repositorio.ufsm.br/bitstream/handle/1/15824/Lic_Computacao_Metodologia-Pesquisa-Cientifica.pdf?sequence=1.

Pereira, R. L. M. R., \& Sampaio, J. P. M. (2019) Estado nutricional e práticas alimentares de idosos do Piauí: dados do Sistema de Vigilância Alimentar e Nutricional-SISVAN Web. Revista Eletrônica de Comunicação, Informação e Inovação em Saúde, Piauí - PI. 13(4), 03-10. 
Research, Society and Development, v. 10, n. 17, e187101724567, 2021

(CC BY 4.0) | ISSN 2525-3409 | DOI: http://dx.doi.org/10.33448/rsd-v10i17.24567

Pillat, A. P., Berlezi, E. M., Jesus, L. B., Schneider, R. H., Beatriz, L., \& Franz, B. (2020), Influência da obesidade nos critérios de classificação de sarcopenia em idosos. Rev. bras. geriatr. gerontol, 23(3), 12-19.

Questionário Whoqol. 2020. Programa de saúde mental Organização Mundial da Saúde. Genebra, OMS. http://www.brasileirosnomundo.itamaraty.gov.br/temas-sociais/questionarios-socio-economicos/programa-de-saude-mental.

Ribeiro C. G \& Ferretti F, \& De Sá C. A. (2017) Quality of life based on level of physical activity among elderly residents of urban and rural areas. Rev Bras Geriatr Gerontol. 20(3), 330-9.

Santos, G. A., Arruda, A. L., Ribeiro, H. S., Correia, H. L., Teixeira, P. S., Neri, S. G. R., Cunha, V. A., Baião, V. M., Melo, W. M. \& Ferreira, A. P. (2018) Descrição do perfil antropométrico, cognitivo e bioquímico de idosos institucionalizados de acordo com o risco de quedas. RPBeCS, 5(9), 01-09.

Silva, J. A. C., Souza, L. E. A. \& Ganassoli, C. (2017) Qualidade de vida na terceira idade: prevalência de fatores intervenientes. Rev Soc Bras Clin Med. julset;15(3),146-169.

Simeão, S. F. A. P., Martins, G. A. L., Gatti, M. A. N., Conti, M. H. S., Vitta, A., \& Marta, S. N. (2018) Estudo comparativo da qualidade de vida de idosos asilados e frequentadores do centro dia. Ciência \& Saúde Coletiva, 23(11), 3923-3934.

Souza, M. S. (2015) Desafios do envelhecimento populacional: como as legislações destinadas aos idosos têm lidado com essa nova demanda? Estudos Interdisciplinares sobre o Envelhecimento, Porto Alegre - RS, 20(1), 159-175.

Souza, Y. P., Bezerra, A. de M., Fabrício, N. P., Tavares, N. B. F., Félix, N. D. C., Viana, M. C. A., \& Soares, A. A. (2018) Qualidade de Vida de Idosos com Obesidade ou Sobrepeso. R bras ci Saúde, 22(2),155-164.

Tavares, D. M. S., Bolina, A. F., Dias, F. P., Ferreira P. S. C., \& Santos, N. M. F. (2018) Excesso de peso em idosos rurais: associação com as condições de saúde e qualidade de vida. Ciênc. Saúde colet. 23(3), 222-243.

Uyeno, D., Lima, M., Nascimento Júnior, J., \& Oiveira, D. (2016) Nível de qualidade de vida dos idosos em instituição de longa permanência - lar dos velhinhos, Maringá/PR. Cinergis, 17(2), 1-6. 